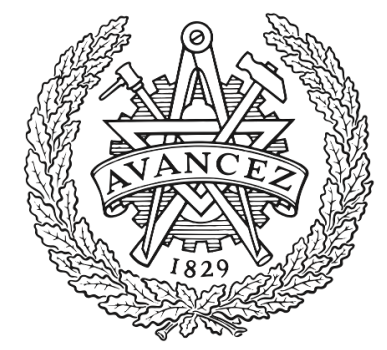

CHALMERS

UNIVERSITY OF TECHNOLOGY

\title{
Switching Dynamics of Dark Solitons in Kerr Microresonators
}

Downloaded from: https://research.chalmers.se, 2023-04-26 15:03 UTC

Citation for the original published paper (version of record):

Nazemosadat Arsanjani, S., Fülöp, A., Helgason, Ò. et al (2019). Switching Dynamics of Dark

Solitons in Kerr Microresonators. Optics InfoBase Conference Papers, paper ef_8_4

N.B. When citing this work, cite the original published paper. 


\title{
Switching Dynamics of Dark Solitons in Kerr Microresonators
}

\author{
Elham Nazemosadat ${ }^{1}$, Attila Fülöp ${ }^{1}$, Óskar B. Helgason ${ }^{1}$, Pei-Hsun Wang ${ }^{2}$, Yi Xuan ${ }^{2}$, Dan E. Leaird ${ }^{2}$, Minghao Qi², \\ Enrique Silvestre ${ }^{3}$, Andrew M. Weiner ${ }^{2}$, and Victor Torres-Company ${ }^{1}$
}

1. Photonics Laboratory, Department of Microtechnology and Nanoscience (MC2), Chalmers University of Technology, Göteborg, Sweden 2. School of Electrical and Computer Engineering, Purdue University, West Lafayette, IN 47907-2035, USA

3. Department of Optics-ICMUV, University of Valencia, 46100 Burjassot, Valencia, Spain

Dissipative Kerr solitons (DKS) are localized structures in optical resonators that arise from a double balance between dispersion and Kerr effect, and linear loss and parametric gain [1]. The periodic nature of DKS corresponds to frequency combs. DKS can be generated in high-Q microresonators for diverse applications, from coherent communications to precision frequency synthesis [1]. Most studies of DKS have focused on microresonator cavities operating in the anomalous dispersion regime, where the waveforms correspond to bright soliton pulses. Coherent microresonator combs can also be formed in the normal dispersion regime [2]. The timedomain waveform corresponds to a localized dark-pulse structure, interpreted as two interlocked switching waves connecting the two branches of the bi-stability curve in continuous-wave-pumped Kerr resonators [2,3]. Each switching wave connects the two branches following an oscillating behavior. These type of Kerr combs are relevant for practical applications because they display unusually high power-conversion efficiency [4,5], but their physical dynamics remain largely unexplored. Here, we report the discovery of deterministic switching of dark pulse Kerr combs, where the number of oscillations that appear between the switching waves can be either increased or decreased one at a time. The switching dynamics observed here have intriguing similarities to the switching behavior of bright temporal solitons in anomalous dispersion microresonators [6], and they indicate that dark pulse Kerr combs arise as a complex interplay of dark solitons circulating in the cavity.

We use a high-Q silicon nitride microresonator pumped with a continuous-wave tunable laser (same as in [5]). The pump laser is tuned into the cavity resonance from the thermally stable blue side with an on-chip power of $\sim 100 \mathrm{~mW}$. Once the comb is generated, distinct steps can be observed in the comb power [Fig. (a)]. Each step corresponds to a coherent comb state [Fig. (b)] that can be accessed in a sequential manner ( $\mathrm{N}=4 \rightarrow 5 \rightarrow 6$ in Fig. (a)). This process was repeated 100 times and similar results were obtained, demonstrating the deterministic nature of the switching dynamics. The measured electrical RF spectra of the corresponding combs show that they are operating in a low-noise state. To understand the physics, we simulate the experimental findings using a modified Ikeda map that takes into account the linear interaction between transverse modes in the cavity. The simulations, shown in Fig. (b), confirm that each discrete jump is associated with the switching of one of the oscillating cycles appearing at the bottom of the dark pulse. The switching transition is reversible by tuning the laser backwards (yellow curve in Fig. (a)). In the backward tuning, the switching occurs over a wider detuning range and ends into what appears to be a single dark soliton state (F). The time domain dynamics leave their footprint in the number of side lobes in the measured spectra (indicated by arrows).

(a)

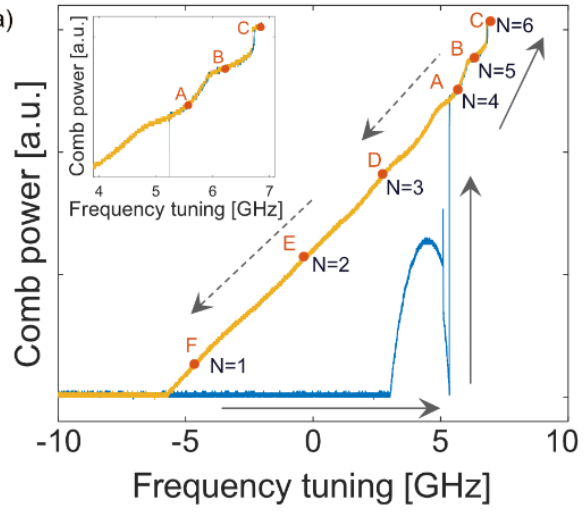

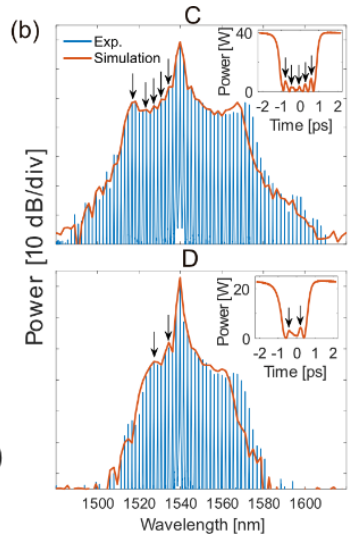
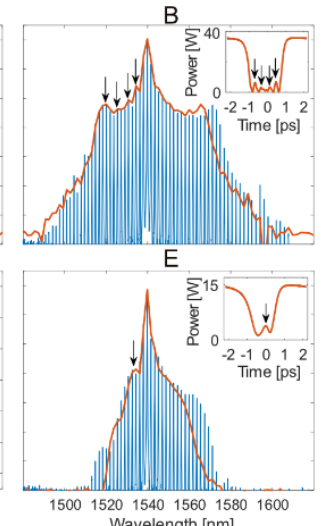
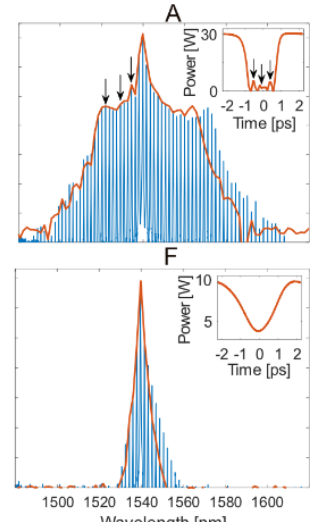

Fig. (a) Measured comb power when the pump is forward (blue) and backward (yellow) tuned. In the forward tuning step like behaviour is observed (zoomed in the inset), indicating switching between multiple dark soliton states. At soliton state $\mathrm{N}=6$, the pump is tuned backwards, and a similar switching behaviour is observed making it possible to switch solitons one by one from $\mathrm{N}=6$ to $\mathrm{N}=1$. (b) Measured (blue) and simulated (red) comb spectra and simulated time domain dark pulses corresponding to each state of (a).

\section{References}

[1] T. J. Kippenberg et al., "Dissipative Kerr solitons in optical microresonators” Science 361, 567 (2018).

[2] X. Xue, et al., "Mode-locked dark pulse Kerr combs in normal-dispersion microresonator,” Nature Photon. 9, 594 (2015).

[3] P. Parra-Rivas, et al., " Origin and stability of dark pulse Kerr combs in normal dispersion resonators," Opt. Lett. 41, 2402 (2016)

[4] X. Xue, et al. "Microresonator Ker frequency combs with high conversion efficiency," Laser \& Photon. Rev. 11, 1600276 (2017).

[5] A. Fülöp, et al., "High-order coherent communications using mode-locked dark-pulse Kerr ...,” Nature Commun. 9, 1598 (2018).

[6] H. Guo, et al. "Universal dynamics and deterministic switching of dissipative Kerr solitons ...,” Nature Phys. 13, 94 (2017). 